\title{
Occurrence of staphylococci in the oral cavities of healthy adults and nasal-oral trafficking of the bacteria
}

\author{
Correspondence \\ Y. Ohara-Nemoto \\ ynemoto@nagasaki-u.ac.jp
}

Received 6 August 2007

Accepted 25 September 2007

\author{
Y. Ohara-Nemoto, ${ }^{1}$ H. Haraga, ${ }^{2}$ S. Kimura ${ }^{2}$ and T. K. Nemoto ${ }^{1}$ \\ ${ }^{1}$ Department of Oral Molecular Biology, Nagasaki University Graduate School of Biomedical \\ Sciences, Nagasaki 852-8588, Japan \\ ${ }^{2}$ Department of Oral Microbiology, Iwate Medical University School of Dentistry, \\ Morioka 020-8505, Japan
}

To investigate a possible peroral route of infective endocarditis (IE), the occurrence of staphylococci in the oral cavity was examined using saliva and supragingival plaque specimens from 56 systemically and periodontally healthy adults aged $22-43$ years old $(27.1 \pm 5.3)$. Nine Staphylococcus species and 334 isolates were identified. In saliva, the total occurrence rate was $83.9 \%$ and the total number of bacteria was $10^{2}-10^{4}$ c.f.u. $\mathrm{ml}^{-1}$. Staphylococcus aureus was the most frequent species (46.4\%), followed by Staphylococcus epidermidis $(41.1 \%)$ and others (Staphylococcus hominis, Staphylococcus warneri, Staphylococcus intermedius, Staphylococcus capitis, Staphylococcus haemolyticus, Staphylococcus lugdunensis and Staphylococcus gallinarum, isolation frequencies ranging in order from 12.5 to $1.8 \%$ ). A similar isolation tendency was observed in supragingival plaque, with a total occurrence rate of $73.2 \%$ and amounts of bacteria ranging from $10^{2}$ to $10^{5}$ c.f.u. $\mathrm{g}^{-1}$. Four common Staphylococcus species (S. aureus, S. epidermidis, S. lugdunensis and S. hominis) were isolated from nasal swab samples taken from the oral staphylococci-positive subjects. Genotyping of all 18 combinations of oral- and nasal-derived isolates by PFGE indicated that identical clones or close relatives were commonly distributed in these two cavities. Since the provision of micro-organisms from the nasal cavity was shown and occurrence rates in the oral cavity were adequate, these results suggest a possible peroral route of staphylococcal IE, as in cases of viridans streptococcal IE.

\section{INTRODUCTION}

Infective endocarditis (IE) is an uncommon but lifethreatening disease of the endocardium of the heart. The most common causative micro-organisms are viridans streptococci, which have been isolated in the blood cultures taken from one-third to one-half of IE patients (Strom et al., 1998; Nakatani et al., 2003; Di Filippo et al., 2006; Ferreiros et al., 2006). Since viridans streptococci comprise a large proportion of the resident oral microflora, their high frequency of isolation from IE patients indicates the functional importance of the tooth-tissue interface as a unique site for these bacteria to enter the bloodstream and potentially infect the heart. Thus much attention has been given to the predisposing conditions of dental procedures and dental hygiene to prevent viridans streptococcal IE, especially in patients with valvular abnormalities (Bayliss et al., 1983; Durack, 1995; Dajani et al., 1997; Tomás Carmona et al., 2002). We in fact recently reported a case of IE caused by Granulicatella elegans originating from the oral cavity (Ohara-Nemoto et al., 2005).
Following viridans streptococci, a high incidence of IE caused by Staphylococcus species has been reported. Among these, Staphylococcus aureus was the most frequently isolated; $20-30 \%$ of whole culture-positive IE cases were due to this species (Strom et al., 1998; Hoen et al., 2002; Nakatani et al., 2003; Cecchi et al., 2004; Ferreiros et al., 2006). Aetiologic Staphylococcus spp. causing IE are assumed to be acquired via a percutaneous route, particularly in cases associated with nosocomial infection and intravenous drug use. However, both of the predisposing conditions and infectious route related to staphylococcal IE are often not specified (Di Filippo et al., 2006). Nakatani et al. (2003) reported cases associated with vascular catheterization $(17.9 \%)$ and dental procedures (14.4\%); however, the main predisposing condition was not identified in more than half of the cases of staphylococcal IE.

Staphylococci are mainly harboured on the skin, as well as skin glands and mucous membranes in humans. Although these micro-organisms are considered to be transiently resident in the oral cavity (Percival et al., 1991; Marsh \& Martin, 1999), during the course of our previous studies we 
have found that the occurrence of Staphylococcus epidermidis was significantly high in saliva from healthy adults (Ikeda et al., 2004). This finding led us to speculate that some of the causative staphylococci in IE originated in the oral cavity. In the present study, we examined the occurrence of Staphylococcus species in saliva and dental plaque specimens from healthy adults. In addition, nasaloral trafficking of these bacterial species was investigated by studying their genotypes using PFGE, to investigate a possible peroral route of IE.

\section{METHODS}

Subjects and clinical specimens. Fifty-six subjects aged 22-43 years old ( $27.1 \pm 5.3$ years; 37 males, 19 females) provided informed consent to participate and were enrolled in this study. The subjects were pre-clinical students and laboratory research staff of Iwate Medical University School of Dentistry, and all were systemically healthy and showed no or negligible pathogenic signs in the periodontal tissues or of dental caries. None of the subjects had received antibiotic medication within the previous 3 months. This study received ethical approval from the Ethics Committees of Iwate Medical University School of Dentistry. Expectorated whole saliva was collected as described previously (Ohara-Nemoto et al., 1997). A supragingival plaque sample was taken from the labial side of the gingival sulcus of the primary or permanently lower left canine with a sterile toothpick, then weighed and immediately suspended in $250 \mu \mathrm{l}$ sterile PBS. Nasal swabs were obtained from the anterior nares of the nose from 18 (32.7 \pm 2.6 years; 12 males, 6 females) of 47 oral staphylococci-positive subjects. The swabs were immediately immersed in $250 \mu$ l sterile PBS.

Culture conditions and species identification. Standard aseptic procedures for sampling and culturing were carefully performed to avoid contamination. Samples of dental plaque and nasal swab in PBS were dispersed by vortex mixing for $30 \mathrm{~s}$, and an aliquot of samples $(100 \mu \mathrm{l})$ at serial dilutions from 1 to $10^{-2}$ was plated onto mannitol salt agar at least in duplicate. After $48 \mathrm{~h}$ at $35^{\circ} \mathrm{C}$ in an aerobic atmosphere, c.f.u. were determined. For species identification, three to six mannitol-fermenting colonies with yellow zones and six to ten non-fermenting colonies with red zones from each sample were subcultured on $5 \%$ sheep-blood agar. Species identification was performed by Gram-staining and a Staphyogram system (Wako Pure Chemical Industries). Colony-direct species-specific PCR was further performed for confirmation of S. aureus (Martineau et al., 1998) and S. epidermidis (Ikeda et al., 2004) isolates, as described previously.

PFGE. PFGE was performed as described previously (Ohara-Nemoto et al., 2005). Briefly, bacterial cells cultured in Todd-Hewitt broth at $35{ }^{\circ} \mathrm{C}$ for $18 \mathrm{~h}$ were harvested and washed with TE $(10 \mathrm{mM}$ Tris/ $\mathrm{HCl}$, $\mathrm{pH}$ 8.0, 1 mM EDTA). Cells were resuspended in Pett IV $(1 \mathrm{M} \mathrm{NaCl}$, $10 \mathrm{mM}$ EDTA, $\mathrm{pH}$ 8.0) to give an $\mathrm{OD}_{600}$ of 0.6. An aliquot of the suspension was mixed with an equal volume of $2 \%$ low-melting agarose solution in TE, to prepare an agarose plug. Agarose plugs were incubated with gentle shaking in lysis solution (10 mM Tris/ $\mathrm{HCl}, \mathrm{pH}$ 8.0, $100 \mathrm{mM}$ EDTA and $25 \%$ glucose) supplemented with $0.5 \mathrm{mg}$ lysozyme $\mathrm{ml}^{-1}, 0.1 \mathrm{mg}$ lysostaphin $\mathrm{ml}^{-1}$ and $10 \mu \mathrm{g}$ RNase A $\mathrm{ml}^{-1}$ at $37{ }^{\circ} \mathrm{C}$ for $16 \mathrm{~h}$. The plugs were incubated in $0.5 \mathrm{M}$ EDTA containing $100 \mu \mathrm{g}$ protease $\mathrm{K} \mathrm{ml}^{-1}$ and $1 \%$ SDS at $50{ }^{\circ} \mathrm{C}$ for $1 \mathrm{~h}$, and then in TE containing $1 \mathrm{mM}$ phenylmethylsulfonyl fluoride at $25{ }^{\circ} \mathrm{C}$ for $1 \mathrm{~h}$. After washing four times with TE, genomic DNA was digested with $20 \mathrm{U} \mathrm{SmaI}$ at $25^{\circ} \mathrm{C}$ for $2 \mathrm{~h}$, then separated with PFGE using a CHEF-DR III apparatus. The initial pulse of $1 \mathrm{~s}$ was increased linearly to $40 \mathrm{~s}$ over $12 \mathrm{~h}$ at $80 \mathrm{~V}$ and $10{ }^{\circ} \mathrm{C}$. Gels were then stained with ethidium bromide and photographed under UV light. Bacterial strain typing to calculate relative coefficients was performed using Fingerprinting II software (Bio-Rad Laboratories).

\section{RESULTS AND DISCUSSION}

\section{Isolation frequencies of oral staphylococci}

Ninety-nine clinical isolates of $S$. aureus and 235 isolates of coagulase-negative staphylococci were identified from saliva and supragingival plaque samples. The isolation frequency of staphylococci was $83.9 \%$ in saliva and $73.2 \%$ in dental plaque, with a total of nine different Staphylococcus species identified (Table 1). The amounts of cultivable staphylococci were $10^{2}-10^{4}$ c.f.u. (ml saliva) ${ }^{-1}$ and $10^{3}-10^{5}$ c.f.u. (g dental plaque) ${ }^{-1}$. The most frequently isolated species in saliva were $S$. aureus $(46.4 \%)$ and $S$. epidermidis $(41.1 \%)$, followed in order by Staphylococcus hominis, Staphylococcus warneri, Staphylococcus intermedius, Staphylococcus capitis and Staphylococcus haemolyticus (12.5-7.1\%). Staphylococcus gallinarum and Staphylococcus lugdunensis were rarely isolated (1/56, $1.8 \%)$. These results indicated that 9 of 15 Staphylococcus species known to be found in humans (Kloos \& Bannerman, 1999) were isolated from the oral cavity. Seven of these species, excluding S. gallinarum and S. lugdunensis, were cultured from the dental plaque samples. The frequency of isolation of staphylococcal species from dental plaque was similar to that from saliva, although S. epidermidis (60.7\%) was more dominant than S. aureus (33.9\%). Staphylococci-positive subjects $(n=47)$ harboured from one to five species $(2.3 \pm 1.0)$ in their samples (some of these results are shown in Table 2). These findings indicated that the occurrence of oral staphylococci was significantly high in systemically and periodontally healthy adult subjects.

Table 1. Occurrence of staphylococci in saliva, dental plaque and nasal samples

\begin{tabular}{|lccc|}
\hline Species & \multicolumn{3}{c|}{$\begin{array}{c}\text { No. of positive subjects } \\
\text { \% isolation frequency) }\end{array}$} \\
\cline { 2 - 4 } & $\begin{array}{c}\text { Saliva } \\
(\boldsymbol{n}=\mathbf{5 6})\end{array}$ & $\begin{array}{c}\text { Plaque } \\
(\boldsymbol{n}=\mathbf{5 6})\end{array}$ & $\begin{array}{c}\text { Nasal } \mathbf{s w a b}^{*} \\
(\boldsymbol{n}=\mathbf{1 8})\end{array}$ \\
\hline S. aureus & $26(46.4)$ & $19(33.9)$ & $8(44.4)$ \\
S. intermedius & $5(8.9)$ & $5(8.9)$ & $0(0)$ \\
S. capitis & $5(8.9)$ & $5(8.9)$ & $3(16.7)$ \\
S. epidermidis & $23(41.1)$ & $34(60.7)$ & $13(72.2)$ \\
S. gallinarum & $1(1.8)$ & $0(0)$ & $0(0)$ \\
S. haemolyticus & $4(7.1)$ & $3(5.4)$ & $1(5.6)$ \\
S. hominis & $7(12.5)$ & $7(12.5)$ & $4(22.2)$ \\
S. lugdunensis & $1(1.8)$ & $0(0)$ & $1(5.6)$ \\
S. warneri & $6(10.7)$ & $5(8.9)$ & $3(16.7)$ \\
Total staphylococci & $47(83.9)$ & $41(73.2)$ & $17(94.4)$ \\
& & & \\
\hline
\end{tabular}

${ }^{\star}$ Nasal swab samples were taken from oral staphylococci-positive subjects. 
Table 2. Staphylococcal species isolated from the oral and nasal cavities

Clinical isolates commonly isolated from oral and nasal cavities are written in bold, and these strains were subjected to PFGE to compare genotypes. ND, Not detected.

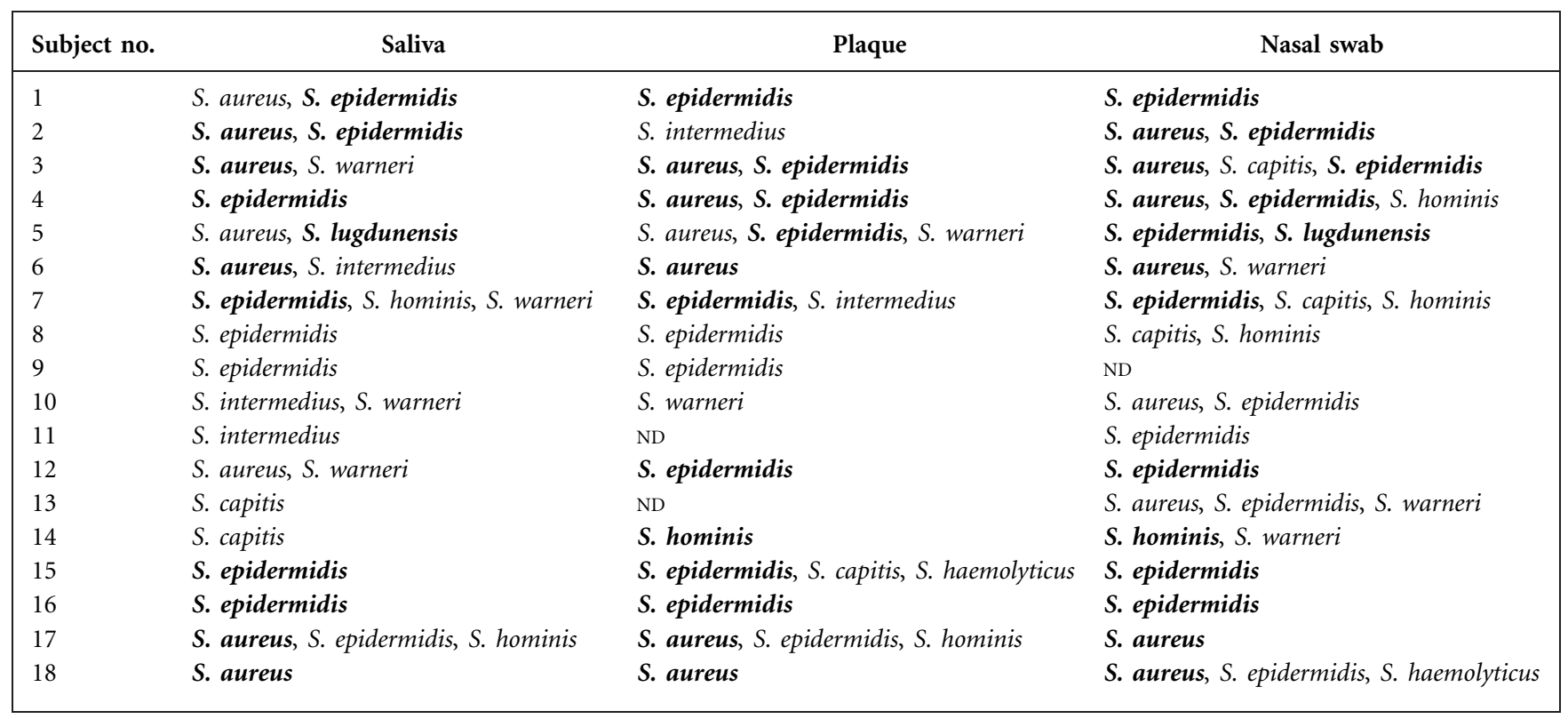

The isolation frequencies in the present report were relatively high compared with previous studies that reported frequencies of $60 \%$ in saliva and $17 \%$ in dental plaque from healthy subjects aged 20-39 years old (Percival et al., 1991), $43 \%$ in plaque from healthy subjects (Murdoch et al., 2004) and 50\% in those with gingivitis and periodontitis (Rams et al., 1990). Although the reason is not clear, larger amounts of oral specimens may be needed for accurate counting of viable staphylococcal cells on selective media. The numbers of cultivable staphylococci from our staphylococci-positive subjects were significantly fewer than those of viridans streptococci by approximately $10^{-2}-10^{-5}$.

\section{Genetic relatedness between oral and nasal staphylococci}

Nasal swab specimens from 18 out of 47 oral staphylococci-positive subjects were cultured for isolation of staphylococci, to investigate nasal-oral trafficking of the bacteria. Swab sampling and cultivation were performed at the same time $(n=3)$ or within 2 weeks after the oral examination $(n=15)$. Consequently, a total of seven different Staphylococcus species were identified in nasal swab specimens (Table 1). Among them, S. epidermidis was predominant $(72.2 \%)$, followed by $S$. aureus $(44.4 \%)$. In 13 of the subjects, S. aureus, S. epidermis, S. hominis and $S$. lugdunensis were commonly isolated from both oral and nasal specimens (Table 2). S. capitis, S. haemolyticus, S. intermedius and $S$. warneri were found in either oral or nasal specimens, probably due to low bacterial numbers.

To compare strain similarities, genotypes of all 18 combinations of the Staphylococcus species (Table 2) consisting of 91 strains commonly found in both the oral and nasal cavities were investigated using PFGE. Representative results are presented for $S$. epidermidis (Fig. 1) and S. aureus and S. lugdunensis (Fig. 2). PFGE patterns of the $S$. epidermidis isolates from the oral samples were nearly identical to those of the nasal-derived isolates. For example, in the case of subject no. 1, the relative coefficient of one strain from saliva, two strains from dental plaque and four strains from nasal swabs was $76.5 \%$. Further, the coefficient of two strains from the oral cavity and two from the nasal cavity was $82.7 \%$ in subject no. 4. As shown in Fig. 2, the PFGE patterns of one of three $S$. aureus isolates from saliva and two plaque-derived isolates from subject no. 3 were apparently identical to their respective nasal-derived isolates (Fig. 2a). In addition, genotypes of oral and nasal-derived S. lugdunensis isolates from subject no. 5 were indistinguishable, with a relative coefficient of $97.0 \%$ (Fig. 2b). The relative coefficients of all 18 cases ranged from 76.5 to $100 \%$. These results, according to the criteria of Tenover et al. (1995), indicate that identical clones or close relatives were commonly distributed in the oral and nasal cavities, and suggest nasaloral trafficking of staphylococci.

Staphylococci are common residents of the nasal flora (Wenzel \& Perl, 1995; Kluytmans et al., 1997; Güçlü et al., 2007), thus they may consistently pass into the mouth. Contamination from skin flora is also possible; however, the PFGE genotyping provided evidence of nasal-oral trafficking of the Staphylococcus species. Alternatively, the number of indigenous staphylococcal strains may be limited in each individual. In addition, a longitudinal sampling and cultivation of saliva, dental plaque and nasal swab were preliminarily performed with subject numbers 6 
(a)

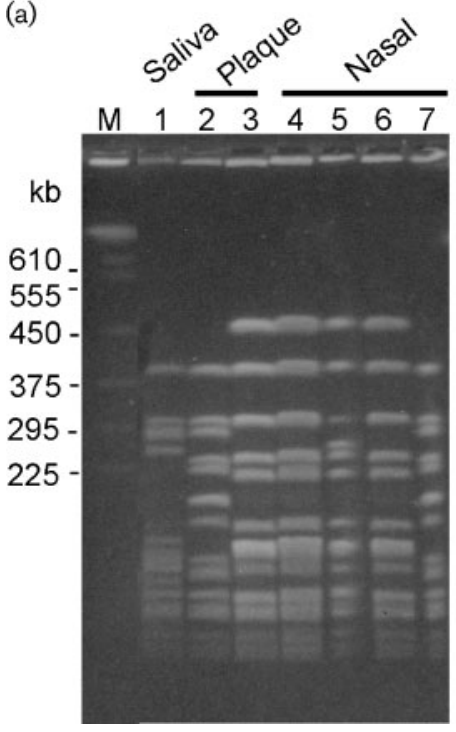

(b)

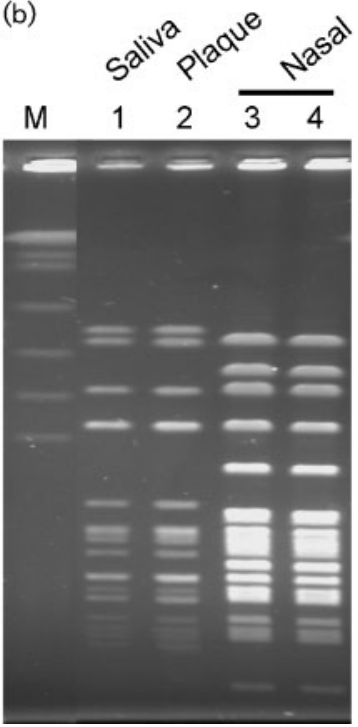

Fig. 1. Genetic relatedness of oral and nasal S. epidermidis isolates. Chromosomal DNA of $S$. epidermidis from saliva, dental plaque and nasal samples from subject no. 1 (a) and no. 4 (b) was digested with Smal and separated using PFGE. The relative coefficients of the strains examined were $76.5 \%$ and $82.7 \%$ (a and $b$, respectively), as calculated with Fingerprinting II software. Lane M, yeast DNA PFGE markers. and 7 over a 2 -month period after the first examination, and we found that $S$. aureus and $S$. epidermidis were continuously isolated from separately collected cultures, respectively. Together, these findings suggest that Staphylococcus species found in the oral cavity are capable of surviving in the oral flora, although these may be continuously provided from the nasal cavity.

S. aureus is the most frequently isolated bacterial species in whole culture-positive IE cases (Hoen et al., 2002; Niwa et al., 2005), even when excluding nosocomial cases and drug users (Strom et al., 1998; Nakatani et al., 2003). S. epidermidis is also a predominant agent for IE (Nakatani et al., 2003; Niwa et al., 2005), and aggressive clinical courses with high mortality IE due to $S$. lugdunensis have been reported (Anguera et al., 2005). All these species were recovered from oral specimens. According to another report (Percival et al., 1991), the rates of occurrence of staphylococci in saliva increase with age. Therefore, considering that most patients suffering from IE are elderly (Hoen et al., 2002; Ferreiros et al., 2006), we think that it is important to assess the peroral route for staphylococcal IE, as in cases with viridans streptococcal IE. In fact, cases of staphylococcal IE after dental extraction and treatments have been previously reported (Bayliss et al., 1983; Etienne et al., 1986). The predisposing condition is occasionally not identified in cases of staphylococcal IE (Nakatani et al., 2003), and thus peroral infection may be involved in those patients. (a)

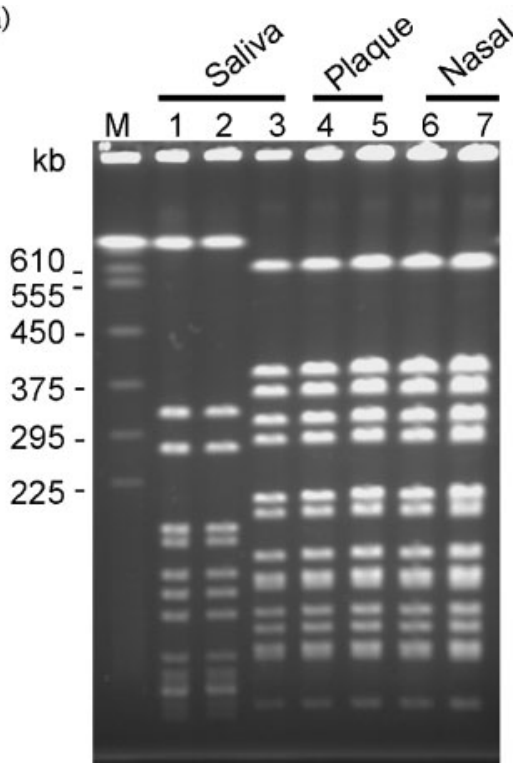

(b)
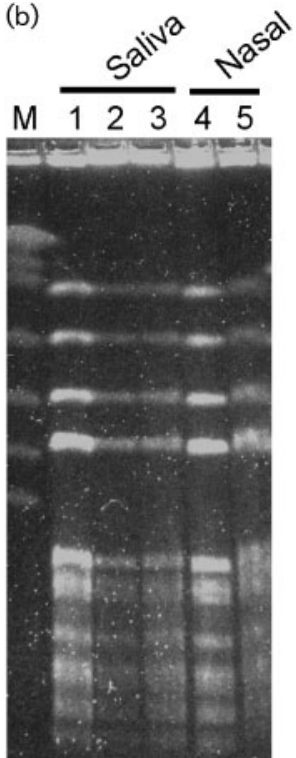

Fig. 2. Genetic relatedness of oral and nasal $S$. aureus and $S$. lugdunensis isolates. Chromosomal DNA of $S$. aureus isolates from saliva, dental plaque and nasal samples from subject no. 3 (a) and S. lugdunensis isolates from subject no. 5 (b) was digested with Smal, then separated using PFGE. The relative coefficients of the strains examined were $100 \%$ (lanes 3-7) and 97.0\% (a and b, respectively). Lane $M$, yeast DNA PFGE markers. 


\section{ACKNOWLEDGEMENTS}

This study was supported in part by a grant-in-aid from the Promotion and Mutual Aid Corporation for Private Schools of Japan.

\section{REFERENCES}

Anguera, I., Del Río, A., Miró, J. M., Matínez-Lacasa, X., Marco, F., Gumá, J. R., Quaglio, G., Claramonte, X., Moreno, A. \& other authors (2005). Staphylococcus lugdunensis infective endocarditis: description of 10 cases and analysis of native valve, prosthetic valve, and pacemaker lead endocarditis clinical profiles. Heart 91, e10.

Bayliss, R., Clarke, C., Oakley, C., Somerville, W. \& Whitfield, A. G. W. (1983). The teeth and infective endocarditis. Br Heart J 50, 506-512.

Cecchi, E., Forno, D., Imazio, M., Migliardi, A., Gnavi, R., Dal Conte, I. \& Trinchero, R. (2004). New trends in the epidemiological and clinical features of infective endocarditis: results of a multicenter prospective study. Ital Heart J 5, 249-256.

Dajani, A. S., Taubert, K. A., Wilson, W., Bolger, A. F., Bayer, A., Ferrieri, P., Gewitz, M. H., Shulman, S. T., Nouri, S. \& other authors (1997). Prevention of bacterial endocarditis. Recommendations by the American Heart Association. JAMA 277, 1794-1801.

Di Filippo, S., Delahaye, F., Semiond, B., Celard, M., Henaine, R., Ninet, J., Sassolas, F. \& Bozio, A. (2006). Current patterns of infective endocarditis in congenital heart disease. Heart 92, 1490-1495.

Durack, D. T. (1995). Prevention of infective endocarditis. N Engl J Med 332, 38-44.

Etienne, J., Fleurette, J., Ninet, J. F., Favet, P. \& Gruer, L. D. (1986). Staphylococcal endocarditis after dental extraction. Lancet 2, 511-512.

Ferreiros, E., Nacinovich, F., Casabé, J. H., Modenesi, J. C., Swieszkowski, S., Cortes, C., Hernan, C. A., Kazelian, L. \& Varini, S. (2006). Epidemiologic, clinical and microbiologic profile of infective endocarditis in Argentina: a national survey. Am Heart J 151, 545-552.

Güçlü, E., Yavuz, T., Tokmak, A., Behçet, M., Karali, E., Öztürk, Ö. \& Egeli, E. (2007). Nasal carriage of pathogenic bacteria in medical students: effects of clinic exposure on prevalence and antibiotic susceptibility. Eur Arch Otorhinolaryngol 264, 85-88.

Hoen, B., Alla, F., Selton-Suty, C., Béguinot, I., Bouvet, A., Briançon, S., Casalta, J. P., Danchin, N., Delahaye, F. \& other authors (2002). Changing profile of infective endocarditis. Results of a 1-year survey in France. JAMA 288, 75-81.

Ikeda, Y., Ohara-Nemoto, Y., Kimura, S., Ishibashi, K. \& Kikuchi, K. (2004). PCR-based identification of Staphylococcus epidermidis targeting gseA encoding the glutamic acid-specific protease. Can $J$ Microbiol 50, 493-498.

Kloos, W. E. \& Bannerman, T. L. (1999). Staphylococcus and Micrococcus. In Manual of Clinical Microbiology, 7th edn, pp. 264282. Edited by P. R. Murray, E. J. Baron, M. A. Pfaller, F. C. Tenover
\& R. H. Yolken. Washington, DC: American Society for Microbiology.

Kluytmans, J., van Belkum, A. \& Verbrugh, H. (1997). Nasal carriage of Staphylococcus aureus: epidemiology, underlying mechanisms, and associated risks. Clin Microbiol Rev 10, 505-520.

Marsh, P. \& Martin, M. V. (1999). The resident oral microflora. In Oral Microbiology, 4th edn, pp. 17-33. Oxford, UK: ButterworthHeinemann Medical.

Martineau, F., Picard, F. J., Roy, P. H., Ouellette, M. \& Bergeron, M. G. (1998). Species-specific and ubiquitous-DNA-based assays for rapid identification of Staphylococcus aureus. J Clin Microbiol 36, 618-623.

Murdoch, F. E., Sammons, R. L. \& Chapple, I. L. (2004). Isolation and characterization of subgingival staphylococci from periodontitis patients and controls. Oral Dis 10, 155-162.

Nakatani, S., Mitsutake, K., Hozumi, T., Yoshikawa, J., Akiyama, M., Yoshida, K., Ishizuka, N., Nakamura, K., Taniguchi, Y. \& other authors (2003). Current characteristics of infective endocarditis in Japan. An analysis of 848 cases in 2000 and 2001. Circ J 67, 901-905.

Niwa, K., Nakazawa, M., Tateno, S., Yoshinaga, M. \& Terai, M. (2005). Infective endocarditis in congenital heart disease: Japanese national collaboration study. Heart 91, 795-800.

Ohara-Nemoto, Y., Tajika, S., Sasaki, M. \& Kaneko, M. (1997). Identification of Abiotrophia adiacens and Abiotrophia defectiva by $16 \mathrm{~S}$ rRNA gene PCR and restriction fragment length polymorphism analysis. J Clin Microbiol 35, 2458-2463.

Ohara-Nemoto, Y., Kishi, K., Satho, M., Tajika, S., Sasaki, M., Namioka, A. \& Kimura, S. (2005). Infective endocarditis caused by Granulicatella elegans originating in the oral cavity. J Clin Microbiol 43, 1405-1407.

Percival, R. S., Challacombe, S. J. \& Marsh, P. D. (1991). Age-related microbiological changes in the salivary and plaque microflora of healthy adults. J Med Microbiol 35, 5-11.

Rams, T. E., Feik, D. \& Slots, J. (1990). Staphylococci in human periodontal diseases. Oral Microbiol Immunol 5, 29-32.

Strom, B. L., Abrutyn, E., Berlin, J. A., Kinman, J. L., Feldman, R. S., Stolley, P. D., Levison, M. E., Korzeniowski, O. M. \& Kaye, D. (1998). Dental and cardiac risk factors for infective endocarditis. A population-based, case-control study. Ann Intern Med 129, 761-769.

Tenover, F. C., Arbeit, R. D., Goering, R. V., Mickelsen, P. A., Murray, B. E., Persing, D. H. \& Swaminathan, B. (1995). Interpreting chromosomal DNA restriction patterns produced by pulsed-field gel electrophoresis: criteria for bacterial strain typing. J Clin Microbiol 33, 2233-2239.

Tomás Carmona, I., Diz Dios, P., Limeres Posse, J., González Quintela, A., Martínez Vázquez, C. \& Castro Iglesias, A. (2002). An update on infective endocarditis of dental origin. J Dent 30, 37-40.

Wenzel, R. P. \& Perl, T. M. (1995). The significance of nasal carriage of Staphylococcus aureus and the incidence of postoperative wound infection. J Hosp Infect 31, 13-24. 